\title{
On the remainder in the Weyl formula for the Euclidean disk
}

\author{
Yves Colin de Verdière *
}

April 21, 2011

\begin{abstract}
We prove a 2-terms Weyl formula for the counting function $N(\mu)$ of the spectrum of the Laplace operator in the Euclidean disk with a sharp remainder estimate $O\left(\mu^{2 / 3}\right)$.
\end{abstract}

\section{Introduction}

Let us denote by $0<\lambda_{1}<\lambda_{2} \leq \cdots$ the eigenvalues for the Dirichlet Laplacian of some bounded connected smooth domain $X$ in the Euclidean plane. It has been shown by Ivrii [11] that the following 2-terms Weyl formula holds under some genericity assumption on the periodic orbits of the associated billiard ball problem: if $N_{X}(\mu)=\#\left\{j \mid \lambda_{j} \leq \mu^{2}\right\}$

$$
N_{X}(\mu)=\frac{|X|}{4 \pi} \mu^{2}-\frac{|\partial X|}{4 \pi} \mu+R(\mu)
$$

with $R(\mu)=o(\mu)$. Moreover, this result is quite optimal: Lazutkin and Terman [13] showed that there is no $\delta>0$ so that an estimate $R(\mu)=O\left(\mu^{1-\delta}\right)$ holds for all smooth convex domains.

Our goal is to get an upper bound for $R(\mu)$ in the case of the Euclidean disk. Our main result ${ }^{1}$ is:

\section{Theorem 1}

$$
N_{\text {disk }}(\mu)=\mu^{2} / 4-\mu / 2+O\left(\mu^{2 / 3}\right) .
$$

*Institut Fourier, Unité mixte de recherche CNRS-UJF 5582, BP 74, 38402-Saint Martin d'Hères Cedex (France); yves.colin-de-verdiere@ujf-grenoble.fr

${ }^{1}$ After having completed this work, we learned from I. Polterovich that the same result has been announced in 1964 by N. V. Kuznecov and B. V. Fedosov in [12]. The method is similar to ours. We give here an independent complete derivation of the needed Van der Corput result 
The proof is based on the explicit expression of the eigenvalues as the squares of the zeros of the Bessel functions $J_{n}$ as well as on some precise asymptotics of these zeros which goes back to Olver (see $[15,5]$ ). This way, we have to study a lattice point problem in some domain with cusps. A rather general lattice point problem was studied by Van der Corput [16], see also [10, 9, 17, 3]. In [4], a similar method was used in order to get a good remainder estimate for some surfaces of revolution. Let us note also that the same remainder estimate holds for the integrable polygonal billiards like the rectangles or the equilateral triangles: this is a direct consequence of the explicit formula for the eigenvalues which reduces the question directly to a lattice point problem for which the Van der Corput's result applies.

\section{The spectrum of the unit disk}

We consider the spectrum of the Euclidean Laplacian $\Delta_{\text {disk }}=-\partial_{x}^{2}-\partial_{y}^{2}$ in the unit disk in $\mathbb{R}_{x, y}^{2}$ with Dirichlet boundary conditions. As it is well known and can be checked by separation of variables, the eigenvalues of $\Delta_{\text {disk }}$ are the squares of the zeros of the Bessel functions $J_{n}, n \in \mathbb{Z}$. Let us recall that

$$
J_{n}(x)=\frac{1}{2 \pi} \int_{-\pi}^{\pi} e^{i(x \sin t-n t)} d t
$$

and that we have the following identities $J_{n}(-x)=(-1)^{n} J_{n}(x), J_{-n}(-x)=$ $J_{n}(x)$. Let us denote by $|n|<x_{1}(n)<x_{2}(n)<\cdots<x_{k}(n)<\cdots$ the positive zeros of $J_{n}$. Then the spectrum of $\Delta_{\text {disk }}$, with multiplicity, is given by

$$
\sigma=\left\{x_{k}(n)^{2} \mid n \in \mathbb{Z}, k=1, \cdots,\right\} .
$$

In order to describe the asymptotics of the zeros of Bessel functions, we introduce the domain $D$ in $\mathbb{R}^{2}$ defined by

$$
D=\{(x, y) \mid-1 \leq x \leq 1, y \leq g(x), y \geq \max (0,-x)\}
$$

with

$$
g(x)=\frac{1}{\pi}\left(\sqrt{1-x^{2}}-x \arccos x\right) .
$$

Let us define $\mathcal{R}=\left\{(n, k-1 / 4) \mid(n, k) \in \mathbb{Z}^{2}\right\}$ and $S=\{(x, y) \mid y \geq$ $\max (0,-x)\}$. Let $F: S \rightarrow \mathbb{R}$ be the function homogeneous of degree 1 which satisfies $F \equiv 1$ on the graph of $g$. The spectrum of the disk is approximately given by $\left\{\lambda_{j} \mid j \in \mathbb{N}\right\} \sim\{F(m) \mid m \in \mathcal{R} \cap S\}$. More precisely, for $n \geq 0$, $x_{k}(n) \sim F(n, k-1 / 4)$, while for $n<0, x_{k}(n) \sim F(n, k+|n|-1 / 4)$. This will reduce our problem to a lattice point problem:

$$
N_{\text {disk }}(\mu) \sim N_{\mathrm{D}}(\mu)=\#\{m \in \mathcal{R} \cap \mu D\} .
$$




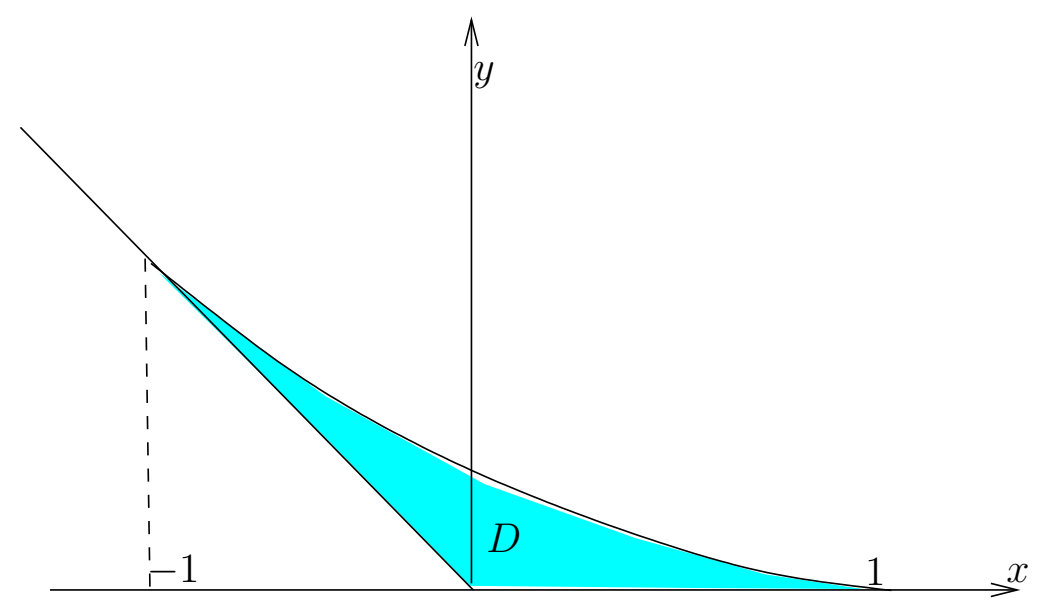

Figure 1: the domain $D$

Remark 1 Let us note that $D$ and $\mathcal{R}$ are invariant by the linear involution $J(x, y)=(-x, y+x)$. This corresponds to the fact that $J_{n}$ and $J_{-n}$ have the same zeros.

In order to complete the argument, we will have to study the lattice point problem (Section 2) and to show how close $N_{\text {disk }}(\mu)$ and $N_{\mathrm{D}}(\mu)$ are (Section $3)$. The first part uses the method of Hlawka, Herz and Randol [10, 9, 17] for studying smooth lattice point problems and the second part is done using Olver's asymptotics for the zeros of Bessel functions: we re-derived it in [5] using the integral representation of the Bessel functions and the general theory of oscillatory integrals associated to versal unfoldings of singularities as explained in [8].

\section{A lattice point problem with a cusp}

Let us denote by $\mathcal{R}$ the lattice $\mathcal{R}:=\left\{(n, k-\beta) \mid(n, k) \in \mathbb{Z}^{2}\right\}$ with $0<\beta<1$. Let us consider a domain $G \subset \mathbb{R}^{2}$ with a cusp: $G=\{(x, y) \mid 0 \leq x \leq 1,0 \leq y \leq$ $g(x)\}$ with $g(x) \sim a(1-x)^{3 / 2}$ with $a>0$ near $x=1$. We consider the weighted lattice point problem defined by the counting function

$$
N_{G, \beta, \chi}(\mu)=\sum_{m=\left(m_{1}, m_{2}\right) \in \mu G \cap \mathcal{R}} \chi\left(m_{2} / m_{1}\right),
$$

with $\chi \in C_{o}^{\infty}(\mathbb{R})$ with $\chi \equiv 1$ near 0 and the support of $\chi$ small enough.

We have the following 2-term Weyl estimate:

Theorem 2 Under the previous assumptions on $G, \beta$ and $\chi$, we have

$$
N_{G, \beta, \chi}(\mu)=\left(\int_{G} \chi(y / x) d x d y\right) \mu^{2}+\left(\beta-\frac{1}{2}\right) \mu+0\left(\mu^{2 / 3}\right) .
$$


Corollary 1 If $D$ is the domain defined in Section 1 and $\beta=1 / 4$, we have

$$
N_{D}(\mu)=\operatorname{Area}(D) \mu^{2}-\frac{\mu}{2}+0\left(\mu^{2 / 3}\right) .
$$

Proof of Corollary 1: we decompose $N_{D}$ into 3 terms: one for each cusp and one inner term using an homogeneous partition of unity. The corollary follows from the previous Theorem for the parts near the cusps and from the classical estimates going back at least to Van der Corput [16] (see also [17, 3]) for the inner part. In order to use Van der Corput estimates $O\left(\mu^{2 / 3}\right)$, we need to check the strict convexity, in fact the non vanishing of the curvature of the graph of $g$ : this comes from the fact that $g^{\prime \prime}(x)=\left(1-x^{2}\right)^{-\frac{1}{2}}>0$.

Proof of Theorem 2: let us denote by $B(m, r)$ the Euclidean ball of center $m$ and radius $r$. We can first replace $\chi(y / x)$ by the smooth function $\chi_{0}(x, y)=$ $\chi(y / x)(1-\phi(x, y))$ with $\phi \in C_{o}^{\infty}$ with support in the ball $B(0, \min (\beta, 1-\beta))$ and $\equiv 1$ near 0 because there is no element of $\mathcal{R}$ in the support of $\phi$. The smooth function $\chi_{0}$ is a classical symbol of degree $0: \partial_{x}^{j} \partial_{y}^{k} \chi_{0}(x, y)=0\left((1+|x|+|y|)^{-(j+k)}\right)$.

Let us give a positive function $\rho \in C_{o}^{\infty}\left(\mathbb{R}^{2}\right)$ with $\operatorname{Support}(\rho) \subset\left\{x^{2}+y^{2}<1\right\}$ and $\int_{\mathbb{R}^{2}} \rho(x, y) d x d y=1$, define $\rho_{\varepsilon}=\rho(. / \varepsilon) / \varepsilon^{2}$ with $\varepsilon=\mu^{-1 / 3}$, and consider

$$
N_{\varepsilon}^{ \pm}(\mu)=\sum_{m \in \mathcal{R}}\left(\chi_{0} \mathbf{1}_{G_{\mu, \varepsilon}^{ \pm}} \star \rho_{\varepsilon}\right)(m)
$$

where

$$
\begin{gathered}
G_{\mu, \varepsilon}^{+}=\{(x, y) \mid 0 \leq x \leq \mu, 0 \leq y \leq \mu g(x / \mu)+2 \varepsilon\} \\
\mathbf{1}_{G_{\mu, \varepsilon}^{-}}=\mathbf{1}_{0 \leq x \leq \mu, 0 \leq y \leq \mu g(x / \mu)-2 \varepsilon}-\mathbf{1}_{0 \leq x \leq \mu, \mu g(x / \mu)-2 \varepsilon \leq y \leq 0} .
\end{gathered}
$$
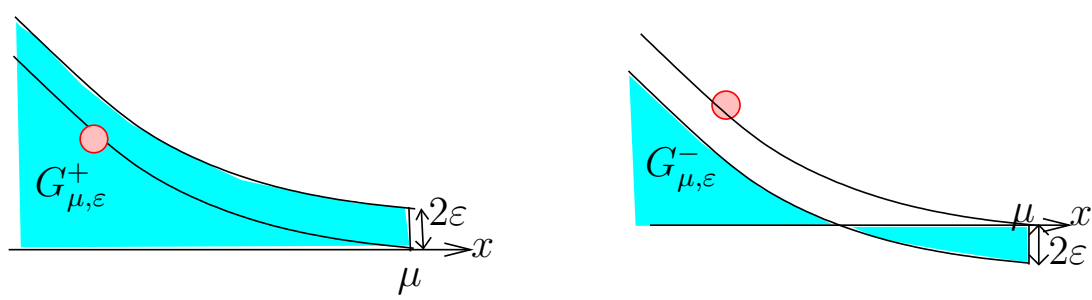

Figure 2: the domains $G_{\mu, \varepsilon}^{ \pm}$

For each $m \notin \mu G$ with $m \in \mathcal{R}, B(m, \varepsilon) \cap G_{\mu, \varepsilon}^{-}=\emptyset$, hence $\left(\mathbf{1}_{G_{\mu, \varepsilon}^{-}} \star \rho_{\varepsilon}\right)(m)=0$ while $\forall(x, y) \in \mathbb{R}^{2}, 0 \leq\left(\mathbf{1}_{G_{\mu, \varepsilon}^{-}} \star \rho_{\varepsilon}\right)(x, y) \leq 1$. Similarly, for each $m \in \mu G \cap \mathcal{R}$, $B(m, \varepsilon) \subset G_{\mu, \varepsilon}^{+}$and $\left(\mathbf{1}_{G_{\mu, \varepsilon}^{+} \star \rho_{\varepsilon}}\right)(m)=1$. Hence,

$$
N_{\varepsilon}^{-}(\mu) \leq N(\mu) \leq N_{\varepsilon}^{+}(\mu)
$$


We will apply Poisson summation formula and use estimates on the Fourier transform of $\mathbf{1}_{G_{\mu, \varepsilon}^{ \pm}}$. Let us denote by

$$
\Phi_{\mu, \varepsilon}^{ \pm}(\xi, \eta)=\int_{\mathbb{R}^{2}} \chi_{0}(x, y) \mathbf{1}_{G_{\mu, \varepsilon}^{ \pm}}(x, y) e^{i(x \xi+y \eta)} d x d y
$$

the Fourier transforms of $\chi_{0}$-times the characteristic function of $G_{\mu, \varepsilon}^{ \pm}$.

The Poisson summation formula applied to the sum (2) gives

$$
N_{\varepsilon}^{ \pm}(\mu)=\int_{\mathbb{R}^{2}} \chi_{0}(x, y) \mathbf{1}_{G_{\mu, \varepsilon}^{ \pm}}(x, y) d x d y+\sum_{(p, q) \in \mathbb{Z}^{2} \backslash 0} \hat{\rho}(2 \pi \varepsilon(p, q)) \Phi_{\mu, \varepsilon}^{ \pm}(2 \pi p, 2 \pi q) e^{-2 \pi i \beta q} .
$$

We need to evaluate $\Phi_{\mu, \varepsilon}^{ \pm}$. We use Green-Riemann formula in order to get integrals on the boundaries. We have the following formulas:

Lemma 1 If $\chi_{0}$ is a smooth classical symbol of degree 0 and

$$
\alpha=\frac{i}{\eta}\left(\chi_{0}(x, y)+\frac{i}{\eta} \partial_{y} \chi_{0}(x, y)-\frac{1}{\eta^{2}} \partial_{y y} \chi_{0}(x, y)\right) e^{i(x \xi+y \eta)} d x
$$

then

$$
d \alpha=\chi_{0}(x, y) e^{i(x \xi+y \eta)} d x \wedge d y+O\left(\eta^{-3}\right) \chi_{1}(x, y) d x \wedge d y,
$$

where $\chi_{1}(x, y) \in L^{1}(d x d y)$.

A similar results holds for

$$
\beta=\frac{1}{i \xi}\left(\chi_{0}(x, y)+\frac{i}{\xi} \partial_{x} \chi_{0}(x, y)-\frac{1}{\xi^{2}} \partial_{x x} \chi_{0}(x, y)\right) e^{i(x \xi+y \eta)} d y
$$

If $|\eta| \leq C|\xi|$, we use

$$
e^{i(x \xi+y \eta)} \chi_{0}(x, y) d x \wedge d y=d \beta+0\left(1 / \xi^{3}\right) \chi_{2} d x \wedge d y,
$$

while, if $|\xi| \leq C|\eta|$, we use

$$
e^{i(x \xi+y \eta)} \chi_{0}(x, y) d x \wedge d y=d \alpha+0\left(1 / \eta^{3}\right) \chi_{1} d x \wedge d y .
$$

We have to estimate the integrals

$$
\int_{\partial G_{\mu, \varepsilon}^{ \pm}} e^{i \xi(x+\nu y)} \chi_{0}(x, y) d y
$$

where $\nu=\eta / \xi$ is bounded (and similar integrals with $\chi_{0}$ replaced by the derivatives of $\chi_{0}$ which are symbols of $<0$ degrees) and

$$
\int_{\partial G_{\mu, \varepsilon}^{ \pm}} e^{i \eta(y+\nu x)} \chi_{0}(x, y) d x
$$


where $\nu=\xi / \eta$ is bounded. We use the upper bounds given in Appendix A for the different parts of the boundaries, using the parametrization of the graph of $y=g(x)$ by $x(t)=1-t^{2} f(t), y(t)=t^{3}$ for $0 \leq t \leq t_{0}$. For example, the main part of the integral on the curved part of $\partial G_{\mu, \varepsilon}^{+}$of $\alpha$ is

$$
\frac{i}{\eta} \int_{0}^{\infty} \chi_{0}(x(t), y(t)) e^{i \mu \eta(y(t)+\nu x(t))} x^{\prime}(t) d t,
$$

to which we apply estimate given in Lemma 8. This gives:

Lemma 2 The following estimates hold:

$$
\Phi_{\mu, \varepsilon}^{ \pm}(0,0)=\mu^{2} \int_{G} \chi(y / x) d x d y+O\left(\mu^{2 / 3}\right)
$$

- For $1 \leq|p| \leq C|q|$,

$$
\Phi_{\mu, \varepsilon}^{ \pm}(2 \pi p, 2 \pi q)=O\left(\frac{\mu^{2}}{(1+\mu\|(p, q)\|)^{3 / 2}}+\frac{1}{|p q|}\right)
$$

- For $p=0, q \neq 0$,

$$
\Phi_{\mu, \varepsilon}^{ \pm}(0,2 \pi q)=\frac{\mu i}{2 \pi q}+O\left(\frac{\mu^{2}}{(1+\mu|q|)^{3 / 2}}\right)
$$

- For $|q| \leq C|p|$,

$$
\Phi_{\mu, \varepsilon}^{ \pm}(2 \pi p, 2 \pi q)=O\left(\frac{\mu^{2}}{(1+\mu\|(p, q)\|)^{3 / 2}}+\frac{1}{\mu^{1 / 3}|p|}\right) .
$$

Let us prove for example the estimate of $\Phi_{\mu, \varepsilon}^{ \pm}(0,2 \pi q)$. The corresponding integral on the boundary splits into 2 parts $\int_{0}^{\mu} . . d x-\int_{0}^{t_{0}} . . d t$. The first part gives the first term. The second part gives, up to constants, $J=q^{-1} \int_{0}^{t_{0}} \exp \left(2 \pi i q \mu t^{3}\right) \chi_{0}(\mu x(t), \mu y(t)) \mu x^{\prime}(t) d t$ which is bounded by $0\left(q^{-1} \mu(q \mu)^{-1 / 2}\right)$ using the estimates of Lemma 8 .

We need also the classical formula:

Lemma 3 For $0<\beta<1$, we have

$$
i \sum_{q \in \mathbb{Z} \backslash 0} e^{-2 \pi i \beta q} / q=2 \pi\left(\beta-\frac{1}{2}\right) .
$$

Theorem 2 follows then from the previous Lemmas and simple evaluations of the sums in the Poisson summation formula (3); using the fact that the Fourier transform of $\rho$ is rapidly decaying, we need the bounds: 
Lemma 4 We have:

$$
\begin{gathered}
\mu^{2} \sum_{(p, q) \in \mathbb{Z}^{2} \backslash 0}(1+\mu\|(p, q)\|)^{-3 / 2}\left(1+\mu^{-1 / 3}\|(p, q)\|\right)^{-N}=O\left(\mu^{2 / 3}\right), \\
\sum_{1 \leq|p| \leq C|q|}|p q|^{-1}\left(1+\mu^{-1 / 3}\|(p, q)\|\right)^{-N}=0\left((\log \mu)^{2}\right) \\
\mu^{2} \sum_{q \neq 0} \mid(1+\mu|q|)^{-3 / 2}\left(1+\mu^{-1 / 3}|q|\right)^{-N}=0\left(\mu^{\frac{1}{2}}\right) \\
\mu^{-1 / 3} \sum_{1 \leq|q| \leq C|p|}|p|^{-1}\left(1+\mu^{-1 / 3}\|(p, q)\|\right)^{-N}=0(1) .
\end{gathered}
$$

Let us check the first upper bound, the others are similar. The first sum is bounded by

$$
C \mu^{\frac{1}{2}} \sum_{(p, q) \in \mathbb{Z}^{2} \backslash 0}\|(p, q)\|^{-3 / 2}\left(1+\mu^{-1 / 3}\|(p, q)\|\right)^{-3 / 2}
$$

which is of the same order as the integral

$$
\mu^{\frac{1}{2}} \int_{0}^{\infty} \frac{r d r}{r^{3 / 2}\left(1+\mu^{-1 / 3} r\right)^{N}}
$$

\section{Spectrum of the disk as a lattice point prob- lem}

Our goal is to prove the following result:

\section{Theorem 3}

$$
N_{\text {disk }}(\mu)=N_{D}(\mu)+O\left(\mu^{2 / 3}\right) .
$$

This will complete the proof of Theorem 1.

Proof.-

The estimate splits into 3 parts: the inner part and the 2 boundary parts. We choose a function $\chi \in C_{o}^{\infty}(]-1,1[,[0,1])$ which is $\equiv 1$ in some large interval $[-1+c, 1-c]$ and split the two numbers to compare as

$$
\begin{gathered}
N_{\text {disk }}(\mu)=N_{\text {disk }}^{1}(\mu)+N_{\text {disk }}^{2}(\mu)+N_{\text {disk }}^{3}(\mu)= \\
=\sum_{x_{k}(n) \leq \mu} \chi(k / n)+\sum_{n \geq 0, x_{k}(n) \leq \mu}(1-\chi(k / n))+\sum_{n<0, x_{k}(n) \leq \mu}(1-\chi(k / n)),
\end{gathered}
$$


and

$$
\begin{gathered}
N_{D}(\mu)=N_{D}^{1}(\mu)+N_{D}^{2}(\mu)+N_{D}^{3}(\mu)= \\
\chi(k / n)+\sum_{n \geq 0,} \sum_{(n, k+\max (0,-n)-1 / 4) \in \mu D}(1-\chi(k / n))+\sum_{n<0,(n, k+|n|-1 / 4) \in \mu D}(1-\chi(k / n)) .
\end{gathered}
$$

We will compare the first terms (the inner parts) in both decompositions and the second terms (the boundary parts). The third ones are similar to the second ones.

The inner part: The zeros $x_{k}(n)$ are given uniformly in any domain $x_{k}(n)>(1+c)|n|$ with $c>0$, by

$$
x_{k}(n)=\left\{\begin{array}{l}
F\left(n, k-\frac{1}{4}\right)+O(1 /(1+k+n)) \text { if } n \geq 0 \\
F\left(n, k+|n|-\frac{1}{4}\right)+O(1 /(1+k+|n|)) \text { if } n<0
\end{array}\right.
$$

This is a consequence of the stationary phase expansion applied to the integral representations (1) of Bessel functions (see [5]). Using the fact that when $F(n, k+\max (0,-n)-1 / 4)$ is close to $\mu,|n|+k$ is of the same order as $\mu$, we get

$$
N_{D}^{1}\left(\mu-\frac{C}{\mu}\right) \leq N_{\text {disk }}^{1}(\mu) \leq N_{D}^{1}\left(\mu+\frac{C}{\mu}\right) .
$$

It follows then from the Van der Corput's remainder estimate $O\left(\mu^{2 / 3}\right)$ for the smooth strictly convex lattice point problems that

$$
N_{\text {disk }}^{1}(\mu)-N_{D}^{1}(\mu)=O\left(\mu^{2 / 3}\right) .
$$

The boundary parts: due to the fact that the zeros of $J_{n}$ and $J_{-n}$ are the same, we discuss only the case $n>0$. We are in a domain where $x_{k}(n)<(1+C) n$. Let us denote by $t_{k}$ the $k$-th zero of the Airy function, we have (see [1])

$$
t_{k}=\left[\frac{3 \pi}{2}\left(k-\frac{1}{4}\right)\right]^{2 / 3}+\varepsilon(k),
$$

with $\varepsilon(k)=O\left(k^{-1}\right)$. From [5], we have the following equation for the zeros $x$ of the Bessel function $J_{n}(x)$ :

$$
\operatorname{Ai}\left(|x|^{2 / 3} \rho(u)\right)+b(u, x)|x|^{-4 / 3} \operatorname{Ai}^{\prime}\left(|x|^{2 / 3} \rho(u)\right)=0,
$$

where $u=(n / x)-1<0, \rho$ is a smooth germ of odd diffeomorphism of $(\mathbb{R}, 0)$ (with $\rho^{\prime}>0$ ) and $b(u, x)$ is a smooth symbol of degree 0 in $x$. We deduce, using the implicit function theorem and the asymptotics of the Airy function, that

$$
x_{k}(n)=n\left(1+\psi\left(\frac{t_{k}}{n^{2 / 3}}\right)\right)+\eta(n, k)
$$


with $\eta(n, k)=O\left(n^{-1}\right), \psi$ smooth, $\psi(0)=0, \psi^{\prime}(0)>0$. This asymptotics is due to Olver [15]. If $(1+C) n>k>(1+c) n>0$ with $0<c<C$, this asymptotics matches with the inner asymptotics via the asymptotics of the $t_{k}$ 's for large $k$ 's.

Let

$$
N_{k}(\mu):=\#\{(n, k-1 / 4) \in \mu D \mid 1 \leq k \leq C n\}
$$

and

$$
N_{k}^{\prime}(\mu):=\#\left\{x_{k}(n) \leq \mu \mid 1 \leq k \leq C n\right\} .
$$

We have the

\section{Lemma 5}

$$
\left|N_{k}(\mu)-N_{k}^{\prime}(\mu)\right| \leq N_{k}\left(\mu+\frac{C}{\mu}\right)-N_{k}\left(\mu-\frac{C}{\mu}\right)+C \mu^{1 / 3} k^{-4 / 3} .
$$

By summing the estimate of the previous Lemma w.r. to $k$ and using the 2-terms asymptotics of $N_{D}^{2}(\mu)$, we get

$$
N_{\text {disk }}^{2}(\mu)-N_{D}^{2}(\mu)=O\left(\mu^{2 / 3}\right) .
$$

Proof of Lemma 5: Let us write $F(x, y):=x\left(1+\psi_{1}\left(y^{2 / 3} / x^{2 / 3}\right)\right)$; we have $N_{k}(\mu)=\#\{F(n, k-1 / 4) \leq \mu \mid 1 \leq k \leq C n\}$ and $N_{k}^{\prime}(\mu)=\#\{F(n, k-1 / 4+$ $\varepsilon(k)) \leq \mu+\eta(k, n) \mid 1 \leq k \leq C n\}$, with $\varepsilon(k)=O(1 / k)$ and $\eta(k, n)=O(1 / n)$. We have, in the range $0<y \leq c x$ with $c$ small enough, $0<a<\partial_{x} F<b$ and $\partial_{y} F=O\left(x^{1 / 3} y^{-1 / 3}\right)$. The Lemma follows by estimating the cardinal of the sets

$$
A_{k}:=\left\{n \mid \mu \leq F(n, k-1 / 4) \leq \mu+\eta(k, n)+C \varepsilon(k) n^{1 / 3} k^{-1 / 3}\right\}
$$

and

$$
A_{k}^{\prime}:=\left\{n \mid \mu-\eta(k, n)-C \varepsilon(k) n^{1 / 3} k^{-1 / 3} \leq F(n, k-1 / 4) \leq \mu\right\} .
$$

We use the fact that if $F(n, k-1 / 4)$ is close to $\mu$ then $n \sim \mu$. We have $A_{k} \subset$ $B_{k} \cup C_{k}$ with $B_{k}:=\{n \mid \mu \leq F(n, k-1 / 4) \leq \mu+O(1 / \mu)\}$ and $C_{k}:=\{n \mid \mu+$ $\left.\eta(k, n) \leq F(n, k-1 / 4) \leq \mu+\eta(k, n)+C \varepsilon(k) n^{1 / 3} k^{-1 / 3}\right\}$. Using the estimate on $\partial_{x} F$, we have $\# C_{k}=O\left(n^{1 / 3} k^{-4 / 3}\right)$

\section{Conclusion and problems}

It would be nice to get similar estimates for other integrable billiards like a circular annulus. The case of ellipse is more difficult and is due to Emile Mathieu [14]: the problem is with the unstable periodic geodesic (the larger diameter). We know now a good approximation of the associated eigenvalues thanks to my works with Bernard Parisse and San Vũ Ngọc ([6, 7]). 


\section{Appendix A: Estimation of some integrals}

We need to get estimates of various integrals corresponding to part of the boundary of the domains $D_{\mu, \varepsilon}^{ \pm}$to be defined in Section 2 .

Let us first recall the following stationary phase estimate:

Lemma 6 Let $f \in C^{\infty}([a, b], \mathbb{C})$ and $\phi \in C^{\infty}([a, b], \mathbb{R})$ so that $\phi$ has only non degenerate critical points, then, if

$$
I(\tau):=\int_{a}^{b} e^{i \tau \phi(t)} f(t) d t
$$

we have $I(\tau)=O\left(\tau^{-\frac{1}{2}}\right)$. If $\phi$ depends smoothly on some parameter $\mu$ so that the non degeneracy assumption holds for $\mu=\mu_{0}$, the same conclusion is true uniformly in some interval $\left|\mu-\mu_{0}\right| \leq c$ with $c$ small enough.

\section{The curved part}

These integrals come when evaluating integrals on the curved part of the domains $D_{\mu, \varepsilon}^{ \pm}$.

Lemma 7 Let us consider the integral

$$
I_{c, A}(\tau)=\int_{0}^{\infty} e^{i \tau\left(\nu t^{3}-t^{2} f(t)\right)} t^{2} g(t) d t
$$

with $g \in C_{o}^{\infty}(\mathbb{R}), f \in C^{\infty}(\mathbb{R}, \mathbb{R})$ with $f(0) \neq 0$ and $|\nu| \leq \nu_{0}<\infty$ with $\nu_{0}$ small enough, then, as $\tau \rightarrow \infty, I_{c, A}(\tau)=O\left(\tau^{-\frac{1}{2}}\right)$ if $\left|\nu_{0}\right|$ is small enough.

This is easy using the stationary phase Lemma 6.

Lemma 8 Let us consider the integral

$$
I_{c, B}(\tau)=\int_{0}^{\infty} e^{i \tau\left(t^{3}-\nu t^{2} f(t)\right)} \operatorname{tg}(t) d t
$$

with $g \in C_{o}^{\infty}(\mathbb{R}), f \in C^{\infty}(\mathbb{R}, \mathbb{R})$, and $|\nu| \leq \nu_{0}<\infty$ with $\nu_{0}$ small enough, then, as $\tau \rightarrow \infty, I_{c, B}(\tau)=O\left(\tau^{-\frac{1}{2}}\right)$.

This is more difficult because the critical point $t=0$ is degenerate for $\nu=0$. We need a

Definition $1 A$ smooth function $f(t, \alpha)$ is in $S^{k}$ if all $t$-derivatives are bounded near $t=\infty$ by $O\left(t^{k}\right)$ uniformly in $\alpha$.

Proof.- 
We will first prove the Lemma for $f \equiv 1$. Let us put $\mu=\nu \tau^{1 / 3}$. We consider 2 cases:

- $|\mu| \leq 1$ : let us make the change $t=w \tau^{-1 / 3}$, we get

$$
I_{c, B}(\tau)=\tau^{-2 / 3} \int_{0}^{\infty} e^{i\left(w^{3}-\mu w^{2}\right)} w g\left(w \tau^{-1 / 3}\right) d w
$$

The critical points of the phase are 0 and $2 \mu / 3$. We split the integral into 2 parts with a smooth partition of unity $1=h+(1-h)$ with $h \in C_{o}^{\infty}\left(\left[0, w_{0}[)\right.\right.$ and $h \equiv 1$ on $[0,1]$. The part containing the critical points is $O(1)$ using an uniform bound for the integrand. For the other part, we introduce $L=1 /\left(3 w^{2}-2 \mu w\right) d / d w$ and integrate by part several times using the formal transpose ${ }^{t} L$ of $L$ and the fact that $w g\left(w \tau^{-1 / 3}\right) \in S^{1}$, so that $\left({ }^{t} L\right)^{N}\left(w g\left(w \tau^{-1 / 3}\right)\right) \in$ $S^{1-2 N}$. If $N \geq 2$, this gives a function which is in $L^{1}(] w_{0},+\infty[)$ uniformly in $\mu$.

- $|\mu| \geq 1$ : we put $t=\nu \sigma$ and get

$$
I_{c, B}(\tau)=\nu^{2} \int_{0}^{\infty} e^{i \mu^{3}\left(\sigma^{3}-\sigma^{2}\right)} \sigma g(\nu \sigma) d \sigma .
$$

We split the integral smoothly and get for the part containing the critical points $O\left(\nu^{2} / \mu^{3 / 2}\right)=O\left(\tau^{-1 / 2}\right)$. For the other part we use $K=\left(1 / 3 w^{2}-2 w\right) d / d w,{ }^{t} K: S^{k} \rightarrow S^{k-2}$ and $\sigma g(\nu \sigma) \in$ $S^{1}$. We pick a factor $\mu^{-3}$ for each integration by parts and get $O\left(\nu^{2} / \mu^{3 N}\right)=O\left(\tau^{-2 / 3}\right)$.

It is clear enough that the proof still works if $f$ is not constant.

\section{The linear parts}

These integrals come when evaluating integrals on the linear parts of the domains $D_{\mu, \varepsilon}^{ \pm}$.

Lemma 9 We have, for $|\eta|=O(|\xi|), I_{v}(\xi, \eta)=\frac{1}{\xi} \int_{0}^{2 \varepsilon} e^{i y \eta} d y=O(\varepsilon /|\xi|)$.

For $\xi=O(|\eta|)$, if

$$
I_{h}(\xi, \eta)=\frac{i}{\eta} \int_{0}^{\mu} e^{i x \xi} d x
$$

then, for $\xi \neq 0, I_{h}(\xi, \eta)=O(1 /|\xi \eta|)$ for $\xi \neq 0$ and $I_{h}(0, \eta)=\frac{i \mu}{\eta}$. 


\section{References}

[1] M. Abramowitz \& I. Stegun, Handbook of Mathematical Functions. National Bureau of Standards Applied Mathematics Series 55, 10th edition (1972).

[2] G.B. Airy, On the Intensity of Light in the neighborhood of a Caustic. Transactions of the Cambridge Philosophical Society 6:379-402 (1838).

[3] Y. Colin de Verdière, Nombre de points entiers dans une famille homothétique de domaines de $\mathbb{R}^{n}$. Ann. Scient. ENS 10 (4):559-575 (1977).

[4] Y. Colin de Verdière, Spectre joint d'opérateurs pseudo-différentiels qui commutent II. Le cas intégrable. Math. Zeitschrift 171:51-73 (1980).

[5] Y. Colin de Verdière, V. Guillemin \& D. Jerison, Singularities of the wave trace near cluster points of the length spectrum. arXiv:1101.0099v1 (2011).

[6] Y. Colin de Verdière \& B. Parisse, Singular Bohr-Sommerfeld rules. Comm. Math. Phys. 205(2):459-500 (1999).

[7] Y. Colin de Verdière \& San Vũ Ngọc, Singular Bohr-Sommerfeld rules for 2D integrable systems. Ann. Sci. École Norm. Sup. (4) 36:1-55 (2003).

[8] V. Guillemin \& D. Schaeffer, Remarks on a paper of D. Ludwig. Bull. of the Amer. Math. Soc. 79:382-385 (1973).

[9] C. S. Herz, On the number of lattice points in a convex set. Amer. J. Math. 84: 126-133 (1962).

[10] F. Hlawka, Über Integrale auf konvexen Körpern I. Monatsh. Math. 54:1-36 (1950).

[11] V. Ivrii, The second term of the spectral asymptotics for a Laplace-Beltrami operator on manifolds with boundary. Functional Analysis and its Applications 14 (2):25-34 (1980).

[12] N. V. Kuznecov \& B. V. Fedosov, An asymptotic formula for the eigenvalues of a circular membrane. Differencial'nye Uravnenija, 1:1682-1685 (1965) (English translation: Differential Equations 1:1326-1329 (1965)) .

[13] V. F. Lazutkin \& D. Ya. Terman, Estimation of the remainder term in the Weyl formula. Functional Analysis and its Applications 15:299-300 (1982).

[14] E. Mathieu, Mémoire sur le mouvement vibratoire d'une membrane de forme elliptique. Journal de Liouville 13(2):137-203 (1868).

[15] F.W.J. Olver, The Asymptotic Expansion of Bessel Functions of Large Order. Phil. Trans. R. Soc. Lond. A 247:328-368 (1954). 
[16] J. G. van der Corput, Über Gitterpunkte in der Ebene. Math. Ann. 81(1): 1-20 (1920).

[17] B. Randol, A lattice-point problem. Trans. Amer. Math. Soc. 121:257-268 (1966).

[18] H. Weyl, Das asymptotische Verteilungsgesetz der Eigenwerte linearer partieller Differentialgleichungen (mit einer Anwendung auf die Theorie der Hohlraumstrahlung). Math. Ann. 71(4):441-479 (1912). 